\section{PHILIP JOSEPH \\ PATRICK KELLY}

Philip Joseph Patrick Kelly LDSI RCSI died peacefully on 13 August 2011 at his home in Woodford Green. He will be sadly missed by his wife Philomena and his two daughters, Geraldine and Marie-Thérèse Hart.

\section{BDA BULLETIN}

\section{- The British Dental}

Association (BDA) has lent its support to the proposed delay on the regulation of general medical practice by the Care Quality Commission (CQC), and renewed its argument that the registration of dentistry should be reconsidered too. The arguments are made in the BDA's response to the Department of Health's consultation on the registration of primary care medical services providers with the $\mathrm{CQC}$. The response highlights the severe policy and administrative difficulties experienced by the CQC.

- The BDA warns that dental public health expertise and budgets must be safeguarded if the Government's proposals for the reform of commissioning and dental services are to succeed. On 26 July the BDA published Dental public health futures which identifies a number of crucial areas in which a failure to protect dental public health in the transition to new NHS structures and systems could create serious problems. The document, which supports the strengthening of public health outlined in the White Paper Healthy lives, healthy people, is available at: http:// www.bda.org/dentists/ policy-campaigns/publichealth-science/public-health/ DPHFutures.aspx

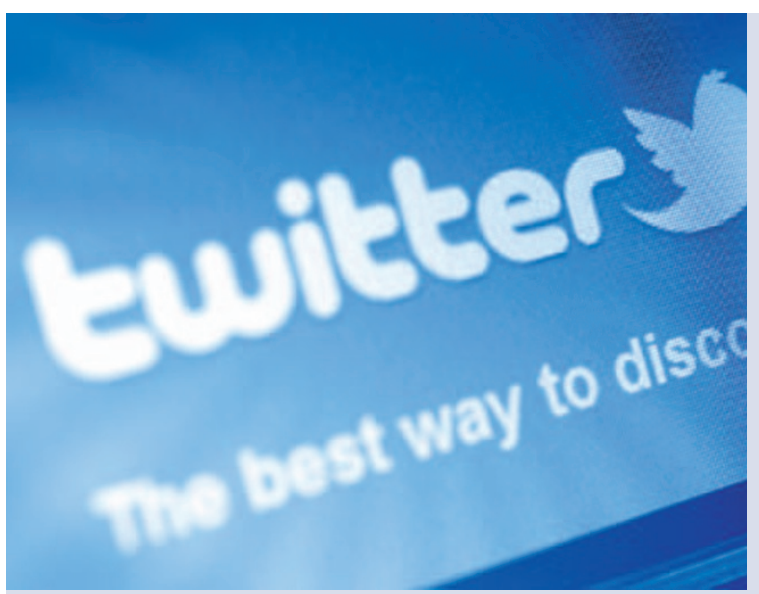

\section{'I'VE GOT \#TOOTHACHE'}

A new study published in the Journal of Dental Research (JDR) demonstrates that users of the social networking website Twitter are using the site to share experiences of toothache and seek advice from other 'tweeters'. Twitter could afford healthcare professionals new ways to communicate with patients.

The study, titled Public health surveillance of dental pain via Twitter, conducted by researchers from the University of California San Francisco (UCSF), investigated the content of Twitter posts meeting search criteria relating to dental pain. A set of 1,000 'tweets' was randomly selected from 4,859 tweets over seven non-consecutive days.
Eighty-three percent of 772 tweets analysed (ambiguous tweets, spam and repeat users were excluded) were primarily categorised as a statement of dental pain; $22 \%$ as an action taken or contemplated; and $15 \%$ as describing an impact on daily activities. Among the actions taken or contemplated, 44\% reported seeing a dentist, 43\% took an analgesic or antibiotic medication and 14\% actively sought advice from the Twitter community.

'This paper highlights the potential of using social media to collect public health data for research purposes,' said $J D R$ Editor-in-Chief William Giannobile. 'Utilising Twitter is an interesting, early state approach with potential impact in the assessment of large sets of population information.

The article can be accessed at: http://jdr.sagepub.com/ content/90/9/1047.abstract

\title{
HOMINIDS SHRANK MOLARS THROUGH COOKING
}

A research team at Harvard University has shown that Homo erectus, Homo neanderthalensis and Homo sapiens may have evolved smaller molars compared with other primates as a result of softening their food with tools and fire.

Extinct hominids of the species Homo erectus (the precursors of man or Homo sapiens), which evolved around 1.9 million years ago, may have cooked and processed their food, the study suggests.

Chris Organ of the Department of Organismic and Evolutionary Biology and his colleagues estimate that humans would spend $48 \%$ of the day eating if we were ordinary primates, when the actual percentage of time humans spend eating each day is $5 \%$.

In a comparative analysis of non-human primates, modern humans, and 14 extinct hominids, the team analysed molar sizes, body masses, DNA and other characteristics to infer when the pattern of reduced eating times began.

The evolution of smaller molars is not explicable by the amount of overall evolution in the jaws and heads of hominids. Softening food allowed for smaller molars and reduced eating time.

The theory that extinct hominids may have cooked their food explains the small teeth, jaws, and guts of modern humans.

1. Organ $C$, Nunn $C L$ Machanda Z, Wrangham R W. Phylogenetic rate shifts in feeding time during the evolution of Homo. Proc Natl Acad Sci USA 2011; DOI: $10.1073 /$ pnas. 1107806108 